\title{
Appendix 1
}

\section{PrEP Background Information and PrEP Anticipated Stigma Scale \\ Presented in Survey}

\section{PrEP Background Information}

Now we would like you to read some background information about PrEP, which will be important for you to think about when you answer the next set of questions:

- PrEP is a daily pill that can be prescribed to HIV-negative individuals to help prevent them from becoming infected with HIV.

- If they take PrEP once a day before they are exposed to HIV (such as through having sex with someone who is HIV-positive), PrEP can be over $90 \%$ effective in preventing them from getting HIV.

- In July of 2012, the U.S. Food and Drug Administration (FDA) approved a medication called Truvada ${ }^{\circledR}$ as the first PrEP medication, stating, "Truvada is approved for use as part of a comprehensive HIV prevention strategy that includes other prevention methods, such as safe sex practices, risk reduction counseling, and regular HIV testing."

- Providers can now prescribe Truvada as a once-a-day pill to individuals who are at risk for getting HIV.

- People taking PrEP will need to take an HIV test and follow up with their medical provider every 3 months.

- Most insurance companies cover the cost of PrEP and there are PrEP financial assistance programs for people who are uninsured.

- Side effects can include upset stomach and dizziness when first starting PrEP. However, they typically go away after the first few weeks, and most people who take PrEP do not notice any side effects at all. 


\section{PrEP Anticipated Stigma Scale}

Remember: PrEP is a daily HIV prevention pill taken by people who are HIV-negative.

Think about what it would be like to take PrEP. Please rate your agreement with the following statements.

Strongly

Disagree

People would assume I

1 slept around if they

knew I took PrEP

People would think I am

2 a bad person if they knew I took PrEP

People would assume I

3 am gay if they knew I took PrEP

People would assume

4 that I was HIV-positive if they knew I took PrEP

I would feel ashamed to

5 tell other people that I was taking PrEP

My sexual partner(s)

6 would approve of me taking PrEP

My family would

7 approve of me taking PrEP

My friends would

8 approve of me taking PrEP

Strongly

Disagree
Disagree

Agree

Strongly

Agree

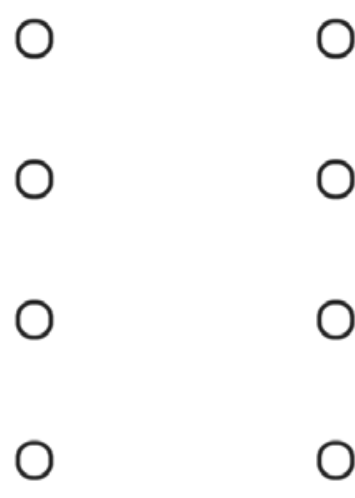

$\mathrm{O}$

O

O

O

O

Disagree

Agree

Strongly

Agree

$\begin{array}{llll}0 & 0 & 0\end{array}$

O $\quad 0$

O $\quad 0$

$\begin{array}{llll}0 & 0 & 0 & 0\end{array}$

$\begin{array}{llll}0 & 0 & 0 & 0\end{array}$ 


\section{PrEP Anticipated Stigma Scale Scoring Instructions}

- For Items 1-5, assign the following values to each response:

o Strongly Disagree $=1$

o $\quad$ Disagree $=2$

o Agree $=3$

o Strongly Agree $=4$

- Reverse score Items 6, 7, and 8 by assigning the following values:

o Strongly Disagree $=4$

o Disagree $=3$

o $\quad$ Agree $=2$

o Strongly Agree $=1$

- Calculate subscale scores by averaging across items:

o PrEP-User Stereotypes: Items 1-5

o PrEP Disapproval by Others: Items 6-8

- Higher subscale scores indicate stronger perceived PrEP-user stereotypes and greater expected PrEP disapproval by others, respectively. 


\section{Appendix 2}

\section{PrEP Anticipated Stigma Scale:}

\section{Subscale Identification and Supplementary Analyses}

\section{Overview of PrEP Anticipated Stigma Scale Development}

In early 2016, eight items were developed based on existing measures of HIV

stigma [e.g., 1] and review of PrEP stigma literature to date [e.g., 2,3]. For example, the item "People would assume I slept around if they knew I took PrEP" was developed based on the HIV stigma item "People assume I slept around because I have HIV" [1]. Items were designed to measure anticipated social stigma surrounding PrEP, including the stereotypical assumptions and (dis)approval by others that participants expected to encounter if they used PrEP. These items were pilot-tested in a sample of HIV-negative, PrEP-inexperienced, sexually active female Planned Parenthood patients who completed the survey between August and November of $2016(n=163)$. The two Planned Parenthood centers from which the pilot sample was recruited did not overlap with the three Planned Parenthood centers from which our present study sample was drawn. Because the measure was intended to capture multiple dimensions of stigma, we performed an exploratory factor analysis on the items as a data-driven approach to delineating subscales in the pilot dataset. We subsequently conducted a confirmatory factor analysis on data collected in the present study $(n=597)$ to further validate this division of subscales.

\section{Exploratory Factor Analysis to Identify Subscales}

With our pilot data set, we performed an exploratory factor analysis with oblique (Promax) rotation and maximum likelihood extraction on the eight scale items using IBM 
SPSS Version 24. All HIV-negative, PrEP-inexperienced, sexually active female Planned Parenthood patients who participated in the pilot survey and completed all PrEP stigma items $(n=163)$ were included. The adequacy of our sample for this analysis was confirmed by a Kaiser-Meyer-Olkin (KMO) value within the "good" range $(\mathrm{KMO}=.80)$ and all $\mathrm{KMO}$ values for individual items $\geq .70$, well above the minimum acceptable value of .50 [4]. Bartlett's test of sphericity indicated sufficiently large interitem correlations to support investigation of underlying factors, $X^{2}(28)=642.40, p<$ .001. The eigenvalue is indicative of the amount of variance that the factor explains. Analysis of initial eigenvalues revealed two factors with eigenvalues greater than Kaiser's criterion of one, which is a commonly used cutoff for determining the number of factors to retain, with values above one considered to account for significant variance [4]. These two factors accounted for $70.36 \%$ of the variance collectively. Table S1 presents all factor loadings. Subscales corresponding to these two factors were constructed from items with factor loadings $>.45$ that did not cross-load on other factors [5], which was all items. These subscales were titled PrEP-User Stereotypes and PrEP Disapproval by Others. Items were coded such that higher scores indicated stronger perceived cultural stereotypes surrounding PrEP users and greater expected disapproval of participants' PrEP use by close others. Items were averaged within subscales to create subscale scores. 
Table S1. Factor Loadings of PrEP Anticipated Stigma Scale Items Determined through Exploratory Factor Analysis of Pilot Data $(n=163)$

\begin{tabular}{|c|c|c|c|}
\hline \multirow[b]{2}{*}{ Subscale } & \multirow[b]{2}{*}{ Item } & \multicolumn{2}{|c|}{ Rotated Factor Loadings } \\
\hline & & 1 & 2 \\
\hline \multirow{5}{*}{$\begin{array}{l}\text { PrEP-User } \\
\text { Stereotypes }\end{array}$} & 1 People would assume I slept around if they knew I took PrEP. & 0.83 & -0.03 \\
\hline & 2 People would think I am a bad person if they knew I took PrEP. & 0.75 & 0.09 \\
\hline & 3 People would assume I am gay if they knew I took PrEP. & 0.58 & -0.10 \\
\hline & 4 People would assume that I was HIV-positive if they knew I took PrEP. & 0.73 & 0.05 \\
\hline & 5 I would feel ashamed to tell other people that I was taking PrEP. & 0.76 & -0.03 \\
\hline \multirow{3}{*}{$\begin{array}{l}\text { PrEP Disapproval } \\
\text { by Others }\end{array}$} & 6 My sexual partner(s) would approve of me taking PrEP. & -0.01 & 0.75 \\
\hline & 7 My family would approve of me taking PrEP. & 0.03 & 0.91 \\
\hline & 8 My friends would approve of me taking PrEP. & -0.02 & 0.91 \\
\hline \multicolumn{2}{|c|}{ Rotated Sums of Squared Loadings } & 2.84 & 2.39 \\
\hline
\end{tabular}

Note. Pattern matrix of exploratory factor analysis with oblique (Promax) rotation and maximum likelihood extraction. Items that loaded uniquely at $\leq-.45$ or $\geq .45$ were retained.

\section{Confirmatory Factor Analysis to Validate Subscales}

Confirmatory factor analysis was performed on data from the present study ( $n=597)$ to test the goodness of fit of a two-factor model using IBM SPSS Amos 20.0. All indicator variables loaded significantly on the two latent constructs, PrEP-User Stereotypes (5 items; Cronbach's $\alpha=0.87$ ) and PrEP Disapproval by Others (3 items; $\alpha=0.91), X^{2}(19)$ $=45.00, p=.001$. All other observed indices used to determine model fit were within the acceptable range: The comparative fit index (CFI), Tucker-Lewis index (TLI), and incremental fit index (IFI) had values greater than .95 and the root mean square error of approximation (RMSEA) had a value below .05 [6,7]. Table S2 presents standardized regression weights of each item and Table S3 presents a summary of fit indices for the final two-factor model. 
Table S2. Standardized Regression Weights for Two-Factor Model

\begin{tabular}{|c|c|c|}
\hline Subcale & Item & $\begin{array}{c}\text { Standardized Regression } \\
\text { Weights }\end{array}$ \\
\hline \multirow{5}{*}{$\begin{array}{l}\text { PrEP-User } \\
\text { Stereotypes }\end{array}$} & 1 People would assume I slept around if they knew I took PrEP. & 0.81 \\
\hline & 2 People would think I am a bad person if they knew I took PrEP. & 0.82 \\
\hline & 3 People would assume I am gay if they knew I took PrEP. & 0.61 \\
\hline & $\begin{array}{l}4 \text { People would assume that I was HIV-positive if they knew I took } \\
\text { PrEP. }\end{array}$ & 0.81 \\
\hline & 5 I would feel ashamed to tell other people that I was taking PrEP. & 0.75 \\
\hline \multirow{3}{*}{$\begin{array}{l}\text { PrEP Disapproval } \\
\text { by Others }\end{array}$} & 6 My sexual partner(s) would approve of me taking PrEP. & 0.88 \\
\hline & 7 My family would approve of me taking PrEP. & 0.86 \\
\hline & 8 My friends would approve of me taking PrEP. & 0.90 \\
\hline
\end{tabular}

Table S3. Confirmatory Factor Analysis Goodness-of-Fit Indices

\section{Supplementary Analyses}

Means and standard deviations of individual PrEP Anticipated Stigma Scale items, inter-item correlations, and correlations between scale items and PrEP uptake indicators are displayed in Table S4. Inter-item correlations were all positive and significant within subscales. Correlations between PrEP stigma items and PrEP uptake indicators were largely negative and significant. 


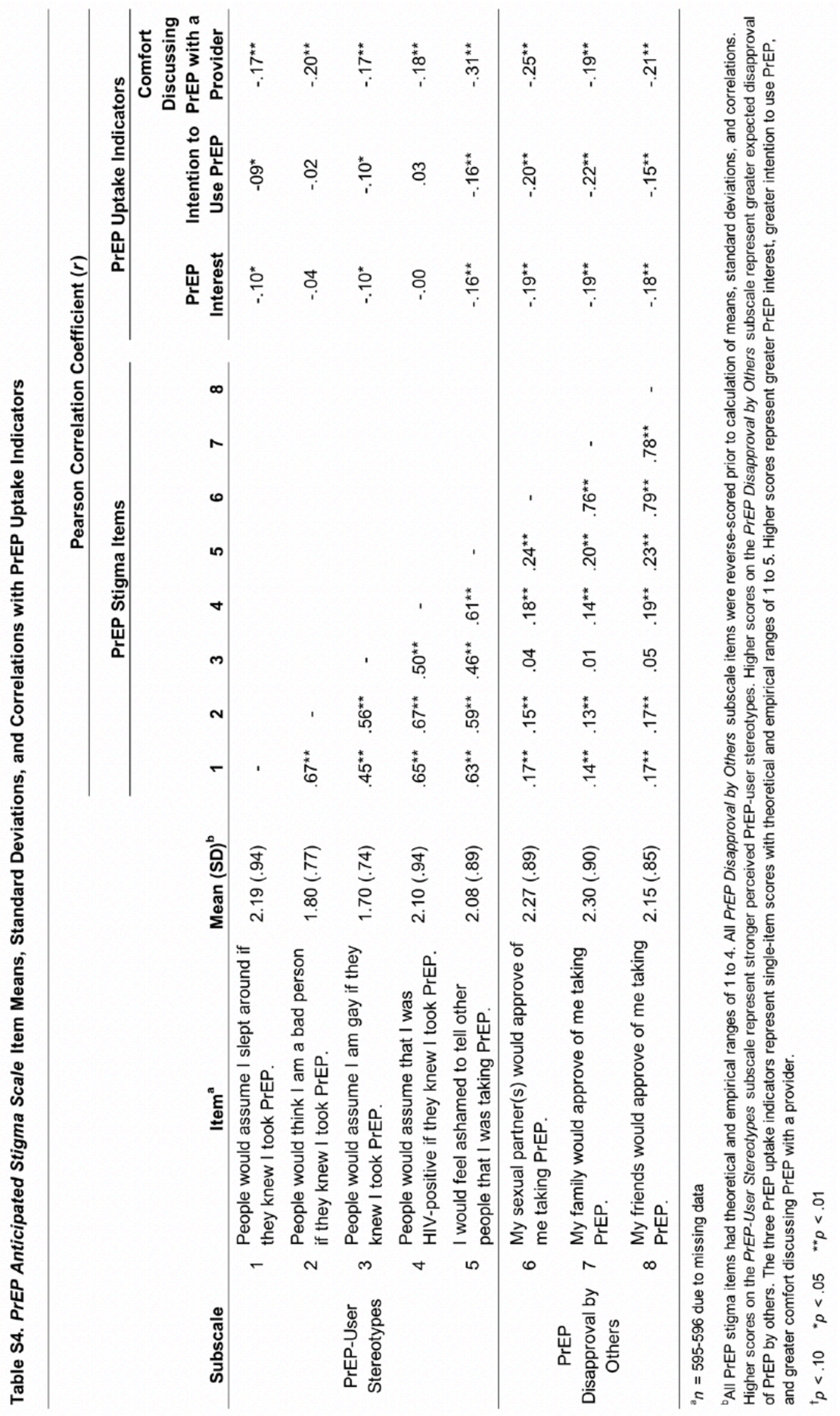




\section{Supplemental Digital Content References}

1. Sayles JN, Wong MD, Kinsler JJ, et al. The association of stigma with selfreported access to medical care and antiretroviral therapy adherence in persons living with HIVIAIDS. J Gen Intern Med 2009,24:1101-1108.

2. Mutchler MG, McDavitt B, Ghani MA, et al. Getting PrEPared for HIV prevention navigation: Young Black gay men talk about HIV prevention in the biomedical era. AIDS Patient Care STDS 2015,29:490-502.

3. Golub SA, Gamarel KE, Surace A. Demographic differences in PrEP-related stereotypes: Implications for implementation. AIDS Behav 2017,21:1229-1235.

4. Field A. Discovering Statistics Using SPSS, $3^{\text {rd }}$ Ed. Thousand Oaks: SAGE Publications Inc.; 2009.

5. Hair JFJ, Anderson RE, Tatham RL, et al. Multivariate Data Analysis, $5^{\text {th }}$ Ed. Dehli: Pearson Education, Inc.; 1998.

6. Schreiber JB, Stage FK, King J, et al. Reporting structural equation modeling and confirmatory factor analysis results: A review. J Educ Res 2006,99:323-337.

7. Sivo SA, Fan X, Witta EL, et al. The search for "optimal" cutoff properties: Fit index criteria in structural equation modeling. J Exp Educ 2006,74:267-288. 